\title{
Ambulatory Anesthesia
}

Editor

MICHAEL T. WALSH

\section{ANESTHESIOLOGY CLINICS}

www.anesthesiology.theclinics.com

Consulting Editor

LEE A. FLEISHER

June 2019 • Volume 37 - Number 2 